\title{
Néel-type skyrmions and their current-induced motion in van der Waals ferromagnet-based heterostructures
}

\author{
Tae-Eon Park $\odot,{ }^{1}$ Licong Peng, ${ }^{2}$ Jinghua Liang, ${ }^{3}$ Ali Hallal, ${ }^{4}$ Fehmi Sami Yasin, ${ }^{2}$ Xichao Zhang, ${ }^{5}$ Kyung Mee Song, ${ }^{1}$ \\ Sung Jong Kim, ${ }^{1,6}$ Kwangsu Kim,,${ }^{1,7}$ Markus Weigand,${ }^{8}$ Gisela Schütz, ${ }^{9}$ Simone Finizio, ${ }^{10}$ Jörg Raabe, ${ }^{10}$ Karin Garcia, ${ }^{11}$ \\ Jing Xia, ${ }^{5}$ Yan Zhou, ${ }^{5}$ Motohiko Ezawa, ${ }^{12}$ Xiaoxi Liu, ${ }^{13}$ Joonyeon Chang, ${ }^{1,14}$ Hyun Cheol Koo, ${ }^{1,6}$ Young Duck Kim, ${ }^{15}$ \\ Mairbek Chshiev, ${ }^{4}$ Albert Fert, ${ }^{11,16}$ Hongxin Yang,,${ }^{3, *}$ Xiuzhen Yu, ${ }^{2, \dagger}$ and Seonghoon Woo ${ }^{1,17, \$}$ \\ ${ }^{1}$ Center for Spintronics, Korea Institute of Science and Technology, Seoul 02792, Korea \\ ${ }^{2}$ RIKEN Center for Emergent Matter Science, Wako 351-0198, Japan \\ ${ }^{3}$ Ningbo Institute of Materials Technology and Engineering, Chinese Academy of Sciences, Ningbo 315201, China Center of Materials \\ Science and Optoelectronics Engineering, University of Chinese Academy of Sciences, Beijing 100049, China \\ ${ }^{4}$ Université Grenoble Alpes, CEA, CNRS, Spintec, 38000 Grenoble, France \\ ${ }^{5}$ School of Science and Engineering, The Chinese University of Hong Kong, Shenzhen, Guangdong 518172, China \\ ${ }^{6} \mathrm{KU}$-KIST Graduate School of Converging Science and Technology, Korea University, Seoul 02841, Korea \\ ${ }^{7}$ Department of Physics, University of Ulsan, Ulsan 44610, Korea \\ ${ }^{8}$ Helmholtz Center Berlin, Albert Einstein Straße 15, 12489 Berlin, Germany \\ ${ }^{9}$ Max-Planck-Institut für Intelligente Systeme, 70569 Stuttgart, Germany \\ ${ }^{10}$ Swiss Light Source, Paul Scherrer Institut, 5232 Villigen, Switzerland \\ ${ }^{11}$ DIPC and University of the Basque Country, 2018 San Sebastian, Spain \\ ${ }^{12}$ Department of Applied Physics, University of Tokyo, Hongo 7-3-1, Tokyo 113-8656, Japan \\ ${ }^{13}$ Department of Electrical and Computer Engineering, Shinshu University, Wakasato 4-17-1, Nagano 380-8553, Japan \\ ${ }^{14}$ Department of Materials Science \& Engineering, Yonsei University, Seoul 03722, Korea \\ ${ }^{15}$ Department of Physics, Kyung Hee University, Seoul 02447, Korea \\ ${ }^{16}$ Unité Mixte de Physique, CNRS, Thales, Université Paris-Sud, Université Paris-Saclay, Palaiseau 91767, France \\ ${ }^{17}$ IBM T.J. Watson Research Center, 1101Kitchawan Road, Yorktown Heights, New York 10598, USA
}

(Received 13 November 2019; revised 5 February 2021; accepted 8 February 2021; published 5 March 2021)

\begin{abstract}
Since the discovery of ferromagnetic two-dimensional (2D) van der Waals (vdW) crystals, significant interest on such 2D magnets has emerged, inspired by their appealing physical properties and integration with other 2D family for unique heterostructures. In known 2D magnets, spin-orbit coupling (SOC) stabilizes perpendicular magnetic anisotropy down to one or a few monolayers. Such a strong SOC could also lift the chiral degeneracy, leading to the formation of topological magnetic textures such as skyrmions through the Dzyaloshinskii-Moriya interaction (DMI). Here, we report the experimental observation of Néel-type chiral magnetic skyrmions and their lattice (SkX) formation in a vdW ferromagnet $\mathrm{Fe}_{3} \mathrm{GeTe}_{2}$ (FGT). We demonstrate the ability to drive an individual skyrmion by short current pulses along a vdW heterostructure, FGT/h-BN, as highly required for any skyrmion-based spintronic device. Using first principle calculations supported by experiments, we unveil the origin of DMI being the interfaces with oxides, which then allows us to engineer vdW heterostructures for desired chiral states. Our finding opens the door to topological spin textures in the 2D vdW magnet and their potential device application.
\end{abstract}

DOI: 10.1103/PhysRevB.103.104410

\section{INTRODUCTION}

Two dimensional (2D) van der Waals (vdW) crystals have been significantly highlighted as a unique material platform, mainly due to their fascinating physical properties, low-cost fabrication, and high integrability to produce appealing artificial heterostructures [1,2]. The recent addition of magnetic vdW crystals, where intrinsic long-range

\footnotetext{
*Corresponding author: hongxin.yang@nimte.ac.cn

${ }_{\dagger}^{\dagger}$ yu_x@ riken.jp

‡shwoo@ibm.com
}

magnetic orders were observed in $\mathrm{Cr}_{2} \mathrm{Ge}_{2} \mathrm{Te}_{6}$ and $\mathrm{CrI}_{3}$, offered an additional building block to this platform, opening another door to vdW magnet-based spintronics [3-10]. Therefore, significant following interests have emerged and rapidly demonstrated a few key elements for applications, including the magnetoresistance (MR) effects [5,6], gate-tunable room-temperature magnetism [7], and manipulation of magnetization switching by electrical current [11]. However, there are still some challenges for the development of $\mathrm{vdW}$ ferromagnet-based spintronics devices, such as rare candidates of atomic-thickness vdW ferromagnets, poor stability under ambient conditions, and low Curie temperature (usually below room temperature). 
Whereas the long-range magnetic order is often suppressed in $\mathrm{vdW}$ crystals due to thermal fluctuations given by the Mermin-Wagner theorem [12], strong spin-orbit coupling (SOC) in vdW magnets plays an essential role in stabilizing the perpendicular magnetic anisotropy (PMA) and thus overcomes the thermal fluctuations down to a monolayer limit $[4,7]$. In a material with such large SOC and broken inversion symmetry, the antisymmetric exchange interaction, the so called Dzyaloshinskii-Moriya interaction (DMI) [13,14], can emerge and be strong enough to stabilize topological magnetic configurations including skyrmions $[15,16]$. Recent theoretical works have also discussed the emergence of DMI in $\mathrm{vdW}$ magnets with various possible origins, e.g., crystal symmetry or sample boundary, as well as resulting skyrmion stabilization [17-19]. Once established, by taking advantages of other 2D crystals that are stackable and offer unique electrical properties [1,2], this material platform could provide a different route towards skyrmion-based devices that have been challenging with conventional metallic ferromagnets [20,21]. Recently, skyrmionlike spin textures have been observed experimentally in exfoliated vdW ferromagnet materials (e.g., FGT and $\mathrm{Cr}_{2} \mathrm{Ge}_{2} \mathrm{Te}_{6}$ ) and their heterostructures with the magnetic fields [22-26]. However, both Bloch-type and Néel-type skyrmions of the previous results have still been a subject of controversy. In addition, experimental demonstration of their field-driven and current-driven dynamics in $\mathrm{vdW}$ magnets and heterostructures has remained elusive so far.

Here, we experimentally present the observation of Néeltype skyrmions and their ordered crystal structures in a vdW ferromagnetic $\mathrm{Fe}_{3} \mathrm{GeTe}_{2}$-based heterostructures (FGT hereafter). Moreover, we demonstrate the ability to drive skyrmions using nanoseconds current-pulses in 2D heterostructure based on FGT, i.e., FGT/h-BN. Among various types of vdW magnets, FGT exhibits relatively high ferromagnetic transition temperature $\left(T_{c}\right)$, large PMA, and metallic nature that enables efficient charge/spin transport suitable for spintronic applications [7,27]. In this study, we utilize high spatial resolution magnetic imaging techniques, scanning transmission x-ray microscopy (STXM), Lorentz transmission electron microscopy (LTEM), and differential phase contrast microscopy (DPCM) to directly observe magnetic structures in the FGT-based heterostructures. We first show the dynamic generation and stabilization of the skyrmion crystal (SkX, also referred to as skyrmion lattice) state in the FGT flake, where strong pulse-induced thermal fluctuations transform magnetic domains into SkX. We then examine the stability of SkX against thermal fluctuation and magnetic fields, which eventually constitutes an experimental phase diagram of the SkX state. We also present the static generation of magnetic skyrmions and SkX using a tilting magnetic field, where we simultaneously unveil the Néel-type chiral nature of skyrmions stabilized in the SkX state by taking advantage of in-plane magnetization sensitivity in LTEM measurements. Moreover, we present the current-driven motion of skyrmions, where we drive isolated individual skyrmions by short current pulses along a FGT racetrack at speeds approaching a meter per second. Finally, using first principle calculations corroborated by additional DPCM measurements, we demonstrate the presence of significant interfacial DMI at FGT interfaces with oxidized layers, which then allows us to "engineer" the chiral states in the vdW ferromagnetic heterostructure between Bloch and Néel types, by using selective fabrication processes.

\section{RESULTS}

\section{A. Crystal structure and domain configuration}

Figure 1(a) schematically shows the crystal structures of monolayered FGT viewed from $x y$ and $y z$ planes and bilayered FGT exhibiting vdW bonding between monolayers. Each FGT monolayer consists of a $\mathrm{Fe}_{3} \mathrm{Ge}$ covalently bonded slab and two Te layers placed above and underneath the $\mathrm{Fe}_{3} \mathrm{Ge}$, and each layer is separated by a $2.95-\AA$ vdW gap in multilayered stack [28]. Within a $\mathrm{Fe}_{3} \mathrm{Ge}$ slab, two inequivalent $\mathrm{Fe}$ sites exist, $\mathrm{Fe}^{\mathrm{II}}$ (the valence states of $\mathrm{Fe}^{2+}$ ) and $\mathrm{Fe}^{\mathrm{III}}$ (the valence states of $\mathrm{Fe}^{3+}$ ), as indicated in Fig. 1(a). Overall, the reduced bulk crystal symmetry in FGT is known to provide a magnetocrystalline anisotropy induced by strong SOC [29]. For both electrical and transmission microscopy measurements on the same sample, we fabricated Hall-bar-type FGT devices on a 100 -nm-thick $\mathrm{Si}_{3} \mathrm{~N}_{4}$ membrane using soft mechanical exfoliation technique together with $e$-beam lithography and liftoff (see Methods and Supplemental Material Fig. S1 [30] for details). Figure 1(b) shows the cross-sectional view of high-resolution transmission electron microscopy (HRTEM) images of the device, where layered high-crystalline quality FGT is observed [Fig. 1(b), inset]. Note that the FGT layer is sandwiched by two oxidized FGT (O-FGT) due to the sample fabrication under ambient condition, and 5-nm-thick Pt was deposited ex situ as a capping material to prevent further oxidation (see Methods and Supplemental Material Fig. S2 [30] for details). The magnetic hysteresis behaviors of the FGT device were measured using the Hall resistance $\left(R_{x y}\right)$ measurement, where external magnetic field was applied to the out-of-plane direction at controlled temperatures ranging 100-220 K [Fig. 1(c)]. While the $R_{x y}$ consists of a normal Hall resistance $\left(R_{\mathrm{N}}\right)$ and an anomalous Hall resistance $\left(R_{\mathrm{AH}}\right)$, FGT films exhibit a large value of $R_{\mathrm{AH}}$ in $R_{x y}$, which roughly scales with the magnetization $\left(M_{z}\right)$ [7]. Therefore, the square hysteresis loops at $100 \mathrm{~K}$ in Fig. 1(c) corresponds to an out-ofplane magnetic anisotropy, which persists up to $200 \mathrm{~K}\left(T_{c} \sim\right.$ $200 \mathrm{~K})$. It is noteworthy that $R_{x y}$ measurements yield two distinct slopes (sharp and slanted slopes) in the temperature range $100 \mathrm{~K} \leqslant T \leqslant 180 \mathrm{~K}$, and the slanted area becomes more prominent as temperature increases. This area indicates the presence of a multidomain state, where the initially nucleated domains at sharp but incomplete switching propagate across the film, originating from the reduced magnetic anisotropy of FGT at higher temperature due to increased thermal fluctuations. Using this slanted area, we can drive the magnetization into the multidomain state at low temperatures and near zero magnetic fields, as shown in Fig. 1(d). Red and blue curves indicate down-to-up and up-to-down switching at $120 \mathrm{~K}$, respectively. For example, at a down-(up-)magnetization saturation state, increasing (decreasing) magnetic field just enough to generate multidomain states and then subsequently reversing the field drives the overall magnetization into multidomain states near zero magnetic field. This technique was employed to generate multidomains during STXM, which 
(a)

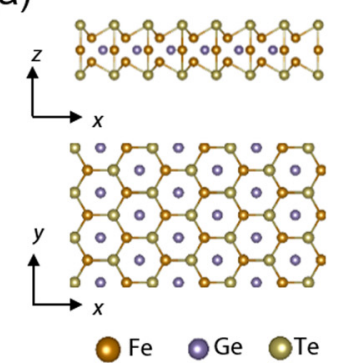

(c)

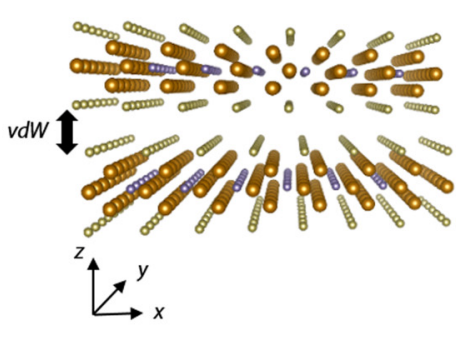

(b)

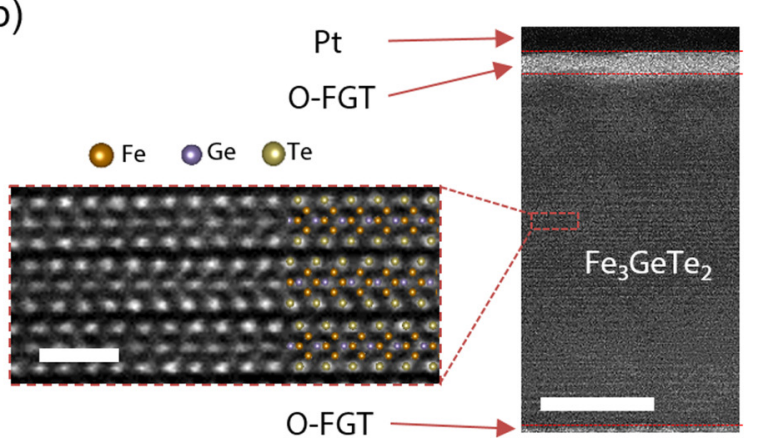

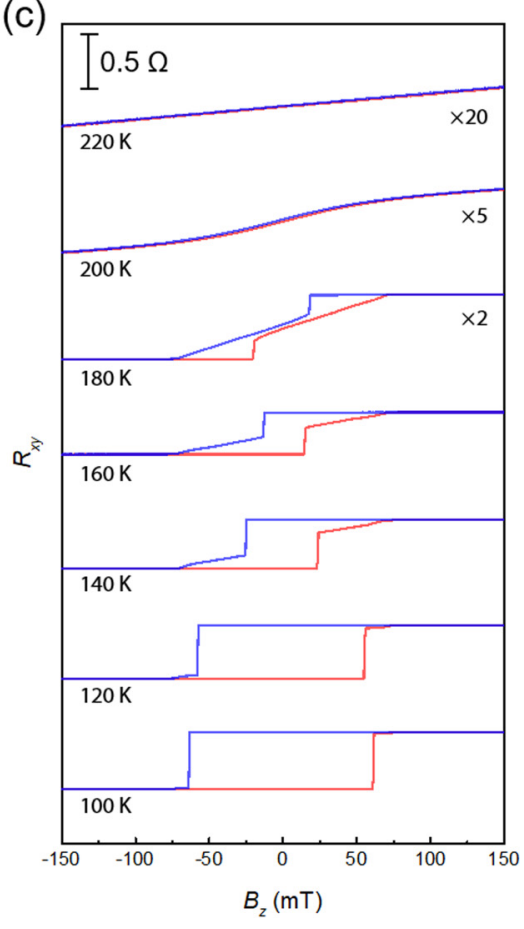

(d)
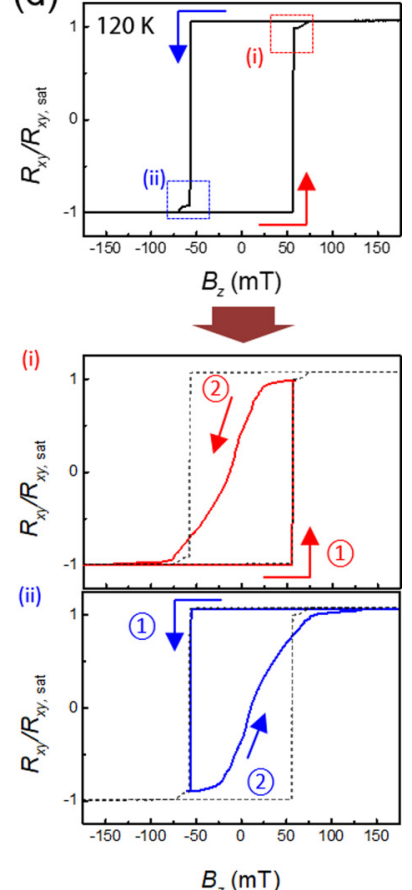

FIG. 1. Crystal structure and the Hall measurement of a van der Waals $\mathrm{Fe}_{3} \mathrm{GeTe}_{2}$. (a) An atomic structure of a Fe $\mathrm{FeTe}_{2}$ (FGT) monolayer (left) and the structure of a FGT bilayer with an interlayer van der Waals (vdW) gap (right). Fe $\mathrm{F}^{\mathrm{III}}$ and Fe $\mathrm{Fe}^{\mathrm{II}}$ represent the two inequivalent Fe sites in the +3 and +2 valence states, respectively. (b) Cross-sectional high-resolution transmission electron microscopy (HRTEM) image of the FGT with a Pt capping Hall-bar device fabricated on 100-nm-thick $\mathrm{Si}_{3} \mathrm{~N}_{4}$ membrane substrate (right, scale bar is $20 \mathrm{~nm}$ ). Oxidized FGT is indicated as O-FGT. The enlarged panel shows the high angle annular dark field (HAADF) image in scanning TEM mode of the red-dashed highlighted area in (b) (left, scale bar is $1 \mathrm{~nm}$ ). (c) Temperature dependent Hall resistance $\left(R_{x y}\right)$ as a function of applied out-of-plane magnetic field $B_{z}$. (d) The measured normalized Hall resistance $\left(R_{x y} / R_{x y, \text { sat }}\right)$ as a function of magnetic field, $B_{z}$, at $120 \mathrm{~K}$, where red and blue dashed rectangular boxes represent the areas of multidomains in the hysteresis loops (left). Bottom two hysteresis loops exhibit the magnetic field sequences used for the generation of multidomains near zero fields from (i) $-B_{z}$ saturation and (ii) $+B_{z}$ saturation, respectively. Black dashes lines are included to show the original full saturation hysteresis loops.

require large moments and multidomain states for high contrast observations.

Figure 2(a) shows the schematic of STXM experimental setup, where the temperature of cooling stage was controlled in the range $100 \mathrm{~K} \leqslant T \leqslant 300 \mathrm{~K}$ using liquid nitrogen $\left(\mathrm{LN}_{2}\right)$ and heat exchanger. The scanning electron microscopy (SEM) image of the measured FGT device with Hall-cross geometry and the electrical circuit diagram is also included in Fig. 2(a) (see Methods for details). The magnetization state of the FGT device was imaged by probing the intensity of transmitted circularly polarized x-ray at the $\mathrm{Fe}$ edge ( $L_{3}$ absorption edge), where x-ray magnetic circular dichroism (XMCD) provides contrasts corresponding to the out-of-plane magnetization. Figure 2(b) shows the magnetic domain configurations in the FGT device as a function of out-of-plane magnetic field $B_{z}$ at $120 \mathrm{~K}$, which confirms strong magnetic contrast observable in FGT from STXM measurements. Note that the alternative field-sweep procedure $\left(B_{z}=+200 \mathrm{mT} \rightarrow-60 \mathrm{mT} \rightarrow 0 \mathrm{mT}\right)$ described in Fig. 1(d) was used to generate the initial magnetic configuration at zero field, $B_{z}=0 \mathrm{mT}$. The dark and bright contrasts in STXM images correspond to downward $\left(-M_{z}\right)$ and upward $\left(+M_{z}\right)$ out-of-plane magnetization direction of Fe atoms in FGT, respectively. With increasing out-of-plane field $B_{z}>$ 0 , the up domains expand while the down domains shrink into narrow domains, vanishing at the saturation field of $B_{z}=+80 \mathrm{mT}$.

\section{B. Dynamic generation and stabilization of SkX}

Having established that multidomain states can be readily stabilized and observed in FGT, we then examined the currentinduced generation of magnetic skyrmions, as summarized in Fig. 3. In our previous study using conventional chiral ferromagnetic multilayers, $\mathrm{Pt} / \mathrm{CoFeB} / \mathrm{MgO}$, we demonstrated that the application of bipolar pulses could transform labyrinth domains with chiral domain walls into multiple skyrmions [31], and the recent study by Lemesh et al. [32] unveiled the mechanism to be current-induced thermal transformation into skyrmions, because the energy barrier towards the global skyrmionic ground state decreases with increasing temperature. To utilize the same technique on the FGT device, we applied a burst of 100 bipolar pulses, where the pulse frequency of $1 \mathrm{MHz}$, the peak-to-peak voltage of $V_{\mathrm{pp}}=2.96 \mathrm{~V}$, and the pulse width of $10 \mathrm{~ns}$ were used at $B_{z}=-40 \mathrm{mT}$ and $120 \mathrm{~K}$. As shown in Fig. 3(a), it is obvious that the bipolar pulse injection transformed the labyrinth random domain state into a multiple circular domain state, where these circular domains turn out to be Néel-type chiral magnetic skyrmions in Fig. 4. It is noted that the labyrinth random domain state 

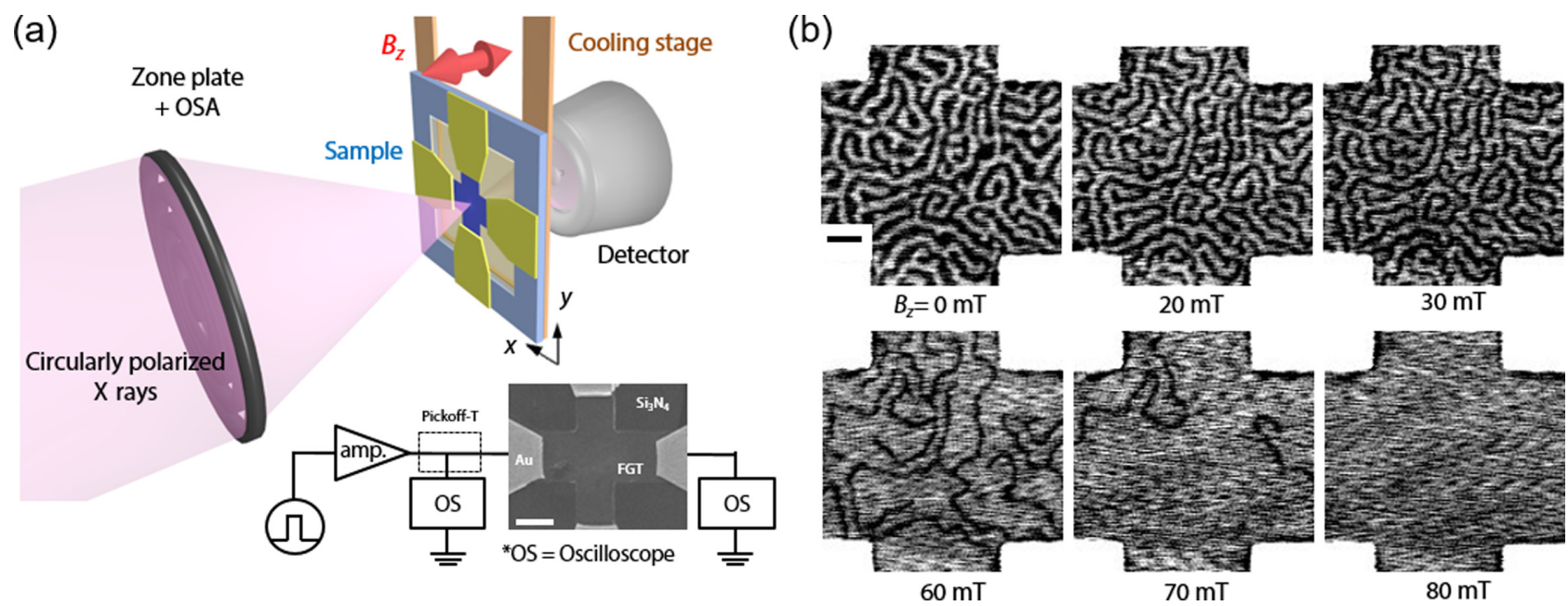

FIG. 2. Microscopy imaging of domain structures using scanning transmission x-ray microscopy. (a) Schematic of scanning transmission $\mathrm{x}$-ray microscopy (STXM) experimental setup used for magnetic domain imaging and simultaneous electrical pulse injections. The inset shows scanning electron microscopy (SEM) image of the measured device with Hall bar geometry. Scale bar, $4 \mu$ m. Two electrode pads on horizontal $x$ axis were used for electrical pulse applications, and oscilloscopes before and after device were used to verify the pulse profiles before and after device, respectively. (b) Exemplary STXM images acquired as a function of increasing magnetic field from $B_{z}=0 \mathrm{mT}$ to $B_{z}=80 \mathrm{mT}$ at $120 \mathrm{~K}$. Dark and bright contrast correspond to magnetization of Fe atoms oriented down $\left(-M_{z}\right)$ and up $\left(+M_{z}\right)$, respectively. Scale bar, $1 \mu \mathrm{m}$.

is maintained when the strong current pulses are applied at zero magnetic field. Thus, the spontaneous transition from the labyrinth random domain state to the skyrmionic state is triggered by both the external magnetic fields and strong current pulses. We performed the same procedure at slightly lower temperature, $100 \mathrm{~K}$, and the consistent transformation into multiple skyrmions is observed and the generated skyrmions remain stable at zero magnetic field, $B_{z}=0 \mathrm{mT}$ [highlighted in a blue-boxed area in Fig. 3(a)]. As was observed in ferromagnetic chiral multilayers, the thermal excitation induced by the bipolar pulses may have opened a path towards the global skyrmionics state [31,32]. We examined and observed the consistent domain transformation in another sample capped by graphite, as shown in Supplemental Material Fig. S3 [30]. This demonstration with graphite capping is significant, as it excludes two possible contributions from Pt: (i) the spin-orbit torques (SOTs) by transmitted spin current caused by the spin-Hall effect (SHE) in Pt [33] and (ii) the DMI contribution from the Pt/O-FGT interface. Additional Hall measurements presented in Supplemental Material Fig. S4 [30] also confirm the negligible influence from capping materials on the magnetic properties of the studied FGT structure. Further analysis reveals that the average size of zero-field skyrmion is $\sim 123 \mathrm{~nm}$ at $120 \mathrm{~K}$, and the size decreases down to $\sim 80 \mathrm{~nm}$ with increased density at $160 \mathrm{~K}$ (see Supplemental Material Fig. S5 for details [30], and Refs. [34-36] therein).

At such a disordered multiskyrmion state at $100 \mathrm{~K}$, we applied alternative positive and negative magnetic fields with increasing magnitude up to $B_{z}= \pm 80 \mathrm{mT}$ with the step of $B_{z}= \pm 10 \mathrm{mT}$, as the application of static fields could annihilate pinned weak skyrmions and rearrange them driven by interskyrmion repulsive forces, leading to the stabilization of the ordered skyrmion state [37,38]. Figure 3(b) shows the zero-field magnetic configuration after the field sweep, and surprisingly, the initial disordered magnetic skyrmions transformed into ordered hexagonal SkX. The inset of Fig. 3(b) presents the enlarged STXM images at a magnetic field, $B_{z}=-80 \mathrm{mT}$, where the ordered SkX state is more clearly observable (a few SkXs are highlighted with blue colors and white lines for guide). As shown in the inset of the enlarged image in Fig. 3(b), the presence of six spots in the fast-Fourier transform (FFT) image also supports the presence of the ordered SkX state in our sample. The symmetry of SkX also agrees with the symmetry observed in noncentrosymmetric B20-type chiral magnets $[15,16]$. After stabilizing the SkX state, we then plotted the experimental phase diagram of magnetic configurations in FGT, based on the real-space STXM measurements as summarized in Fig. 3(c). We observed three magnetic configuration phases: (i) $\mathrm{SkX}$, (ii) the coexistence of SkX and multidomains, and (iii) saturated ferromagnetic states, where the representative STXM images of each state are included in the right panel of Fig. 3(c). It should be noted that, once generated, SkX in FGT can be stabilized at a wide range of magnetic field and temperature. Moreover, the SkX state remains stable at zero magnetic field. Together with the recent discovery of gate-tunable room-temperature magnetization in the same material [7], it might also be possible to harness and manipulate magnetic skyrmions and their lattice at room temperature and zero magnetic fields, which may constitute a major step towards room-temperature skyrmion applications based on vdW magnets.

\section{Lorentz transmission electron microscopy (LTEM) study of SkX}

To deeply understand magnetic configurations observed by STXM measurements, we first performed the LTEM measurement as summarized in Fig. 4 (see Methods for details [30], and Refs. [39-41] therein). Note here that Fresnel-LTEM is useful to detect the in-plane components of Bloch-type spin spirals at defocused modes, whereas it cannot directly observe Néel-type magnetic configurations with zone-axis beam 
(a)

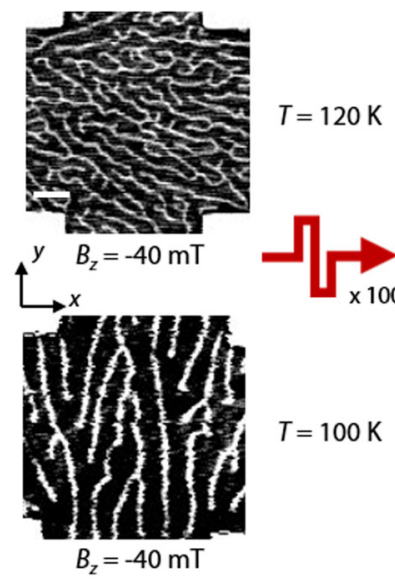

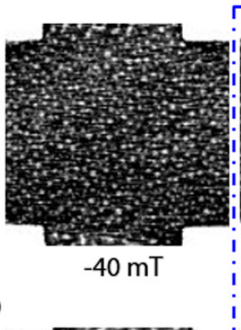
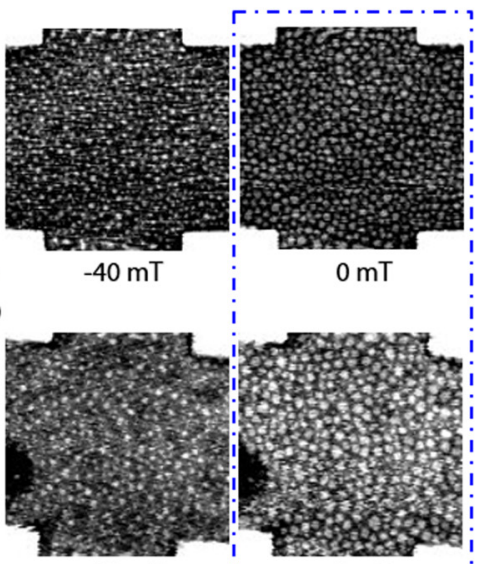

$-40 \mathrm{mT}$

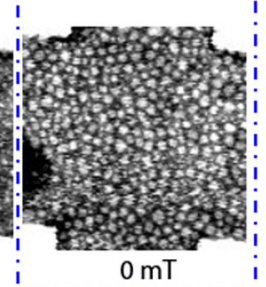

(b)

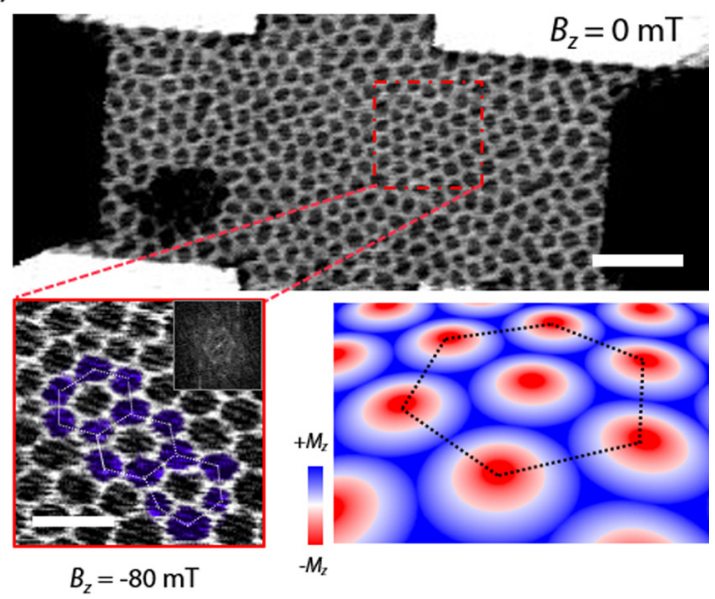

(c)

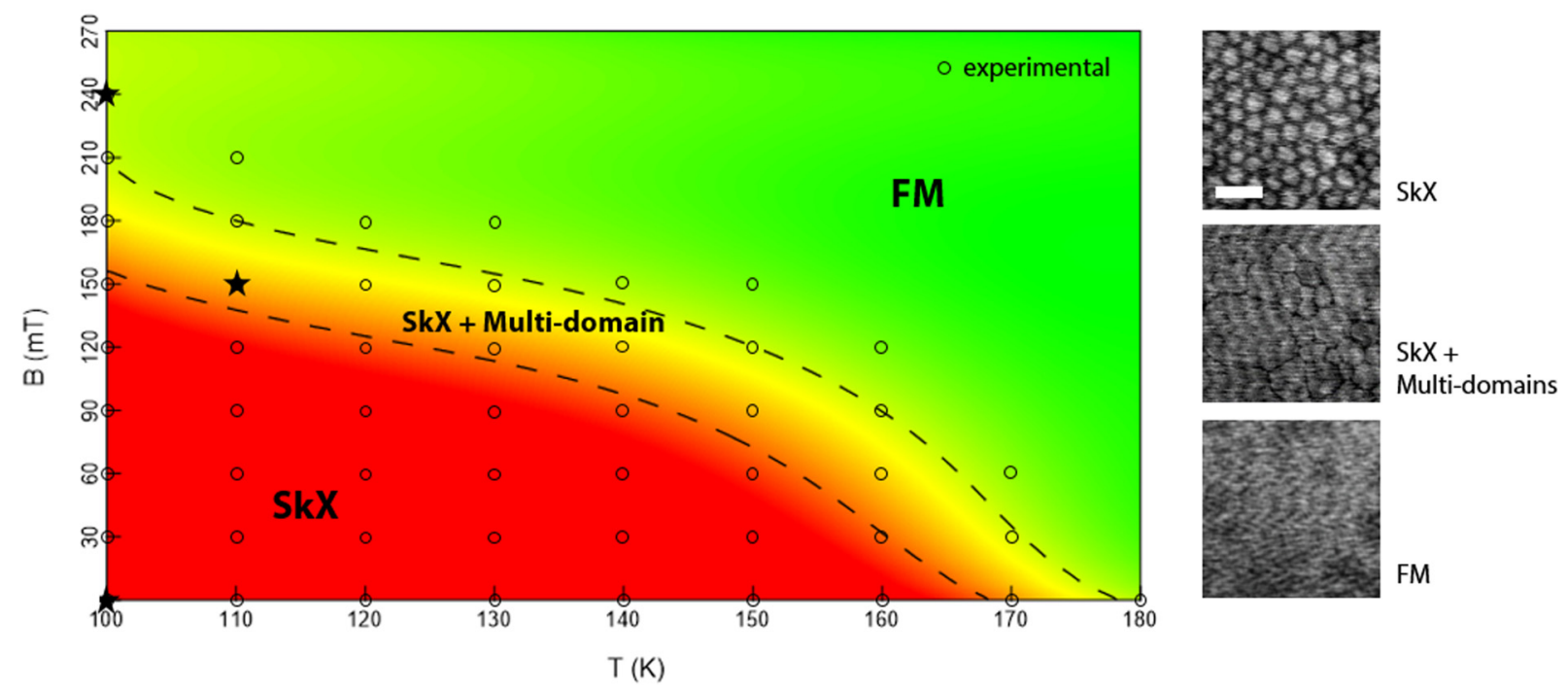

FIG. 3. Generation and stabilization of magnetic skyrmion lattice phase. (a) The two images on the left side were acquired at $B_{z}=-40 \mathrm{mT}$ at 120 and $100 \mathrm{~K}$ after the initial saturation at $B_{z}=+200 \mathrm{mT}$, respectively, where initial labyrinth domain states were stabilized. The right two images at $B_{z}=-40 \mathrm{mT}$ were acquired after the application of bipolar pulse bursts at 120 and $100 \mathrm{~K}$, respectively, and the other two images at $B_{z}=0 \mathrm{mT}$ were acquired after removing magnetic fields. Scale bar, $1 \mu \mathrm{m}$. (b) Representative STXM image of skyrmion crystal (SkX) stabilized over the whole FGT device at $B_{z}=0 \mathrm{mT}$ and $T=100 \mathrm{~K}$. Scale bar, $2 \mu \mathrm{m}$. For clarity, the enlarged image of SkX was obtained at $B_{z}=-80 \mathrm{mT}$. Scale bar, $1 \mu \mathrm{m}$. The hexagonal white lines are drawn to guide eye for the ordered SkX, and inset shows the fast-Fourier transform (FFT) image. The right schematic represents the exemplary magnetic configuration of SkX found in chiral magnets for comparison. Note that skyrmion polarity in (b) $\left(-M_{z}\right.$ core) is different from (a) $\left(+M_{z}\right.$ core), as the initial field-sweep procedure of reversed field direction was used before the pulse application: $B_{z}=-200 \mathrm{mT} \rightarrow+40 \mathrm{mT}$. (c) Experimental phase diagram of magnetic configurations as a function of temperature and magnetic field. Experimentally measured positions are marked with open circles, and star symbols correspond to exemplary images shown on the right side of the phase diagram. Three representative images show each magnetic configuration state: SkX, SkX + multidomains, and saturated ferromagnet $(\mathrm{FM})$. Scale bar, $1 \mu \mathrm{m}$. Black dashed lines in phase diagram are guide to the eyes to indicate the phase boundaries.

irradiation, due to the cancellation of magnetic inductions between electrons and symmetric in-plane magnetic moments with opposite directions projected by Néel-type spin textures $[39,42,43]$. However, when samples are tilted away from the zone axis, the projected configurations of up-down magnetic domains should contribute to the LTEM contrasts at defocused modes, therefore, Néel-type magnetic configurations can be observed [38,39,42,43].

Figure 4(a) first shows in-focus and defocused LTEM images of the FGT sample tilted about $-20^{\circ}$ along the $x$ axis at zero field and $160 \mathrm{~K}$, where dark/bright contrasts are only visible in defocused images. Moreover, as shown in the redboxed areas in the left and right images in Fig. 4(a), underand over-focused LTEM images exhibit the labyrinth domain structures with reserved domain wall contrasts, indicating a multidomain state in the FGT flake at zero field [42-44]. To generate SkX, we then performed the field cooling (FC) of FGT with an oblique magnetic field of $B=-40 \mathrm{mT}$ (the oblique angle is $20^{\circ}$ to the zone axis). Figure 4(b) shows LTEM images observed at $160 \mathrm{~K}$. Noticeably, the FC generated quasistatic (metastable) Néel-type chiral SkX state in the FGT crystal, which is in good agreement with the 
(a)

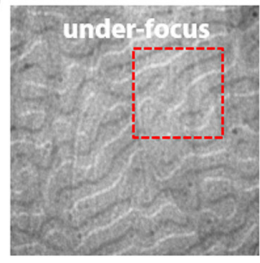

(b)
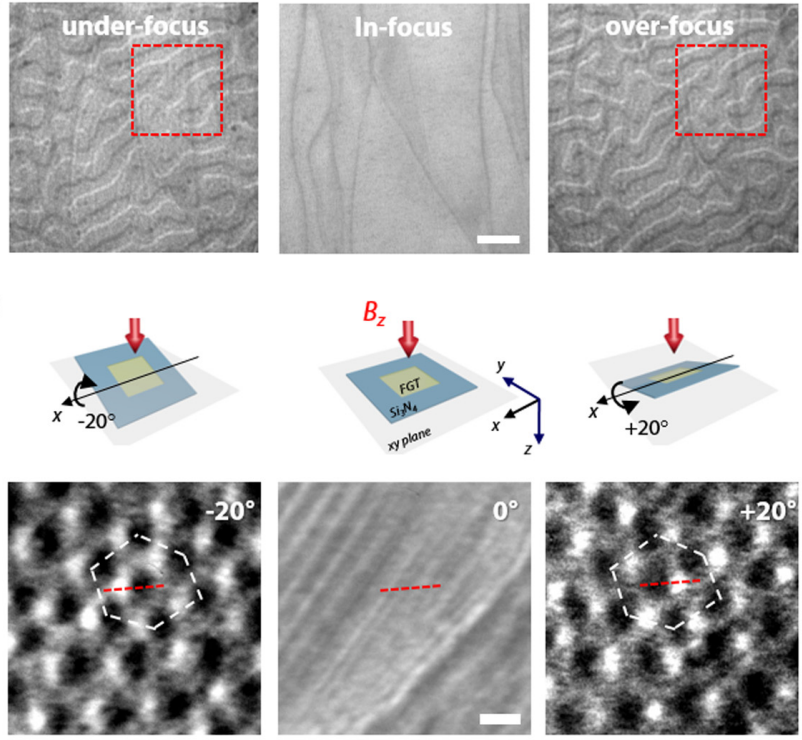

(c)

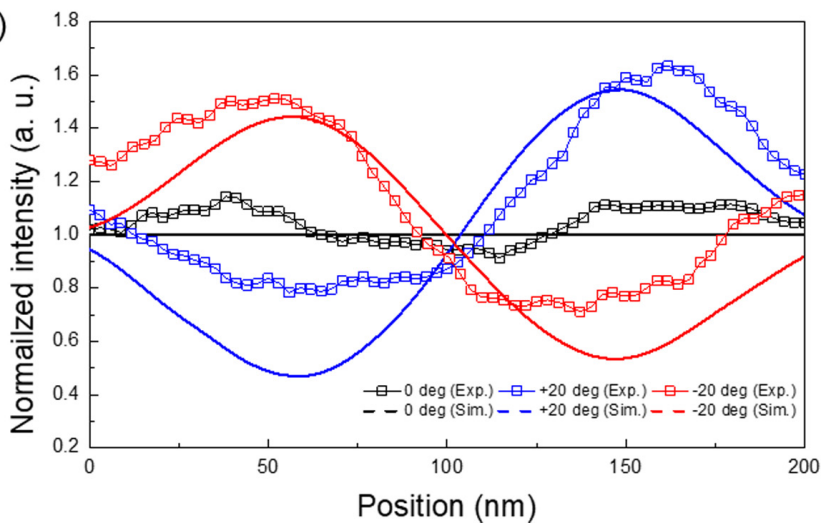

FIG. 4. Lorentz transmission electron microscopy (LTEM) measurements of skyrmion crystals (SkX). (a) LTEM images of magnetic configurations in the FGT flake on off-axis at underfocus (left), in focus (middle), and overfocus (right) acquired at zero field and 160 $\mathrm{K}$, respectively. Scale bar, $500 \mathrm{~nm}$. (b) LTEM images of SkX taken at the flake with tilting angle of $-20^{\circ}$ (left), $0^{\circ}$ (middle), and $20^{\circ}$ (right) with respect to $x$ axis at $B_{z}=-40 \mathrm{mT}$ and $160 \mathrm{~K}$ after the field-cooling (FC) process, respectively. The defocus values are \pm 3 $\mathrm{mm}$ for observing over/underfocus image. The striplike contrasts in (b) at zero-tilt angle indicate bending contours of the flake. White hexagonal-shaped lines are eye guides for the unit of the SkX. Scale bar, $200 \mathrm{~nm}$. Upper panels in (b) are schematic drawings of the flake orientations with respect to the incident electron beam. Solid red lines in (b) guide the line-scan positions for contrast profiles. (c) Experimentally measured (symbols) and simulated (solid lines) contrast profiles across a single skyrmion as shown in (b). Note that the simulated profiles were obtained for a 100-nm-size Néel-type skyrmion.

simulated LTEM results with magnetic configuration and inplane magnetization profiles [Fig. 4(c)] (see Methods and Figs. S6 and S7 for details [30]). Considering that the oblique field applied during the LTEM measurements could have contributed to the in-plane alignment of magnetic moments within FGT domain walls, we have performed LTEM observations of $\mathrm{SkX}$ at zero field after the same FC process as (a)
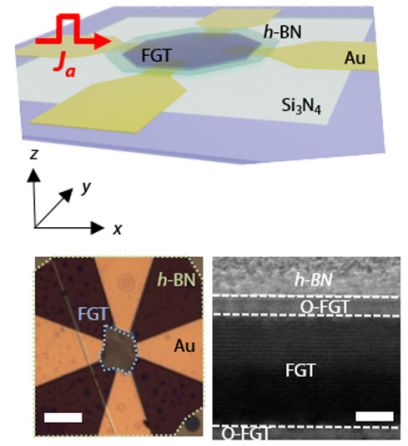

(b)

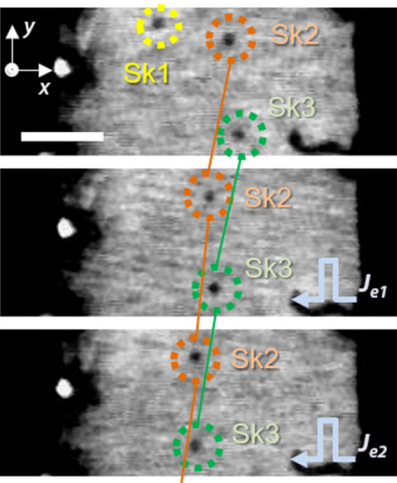

(c)
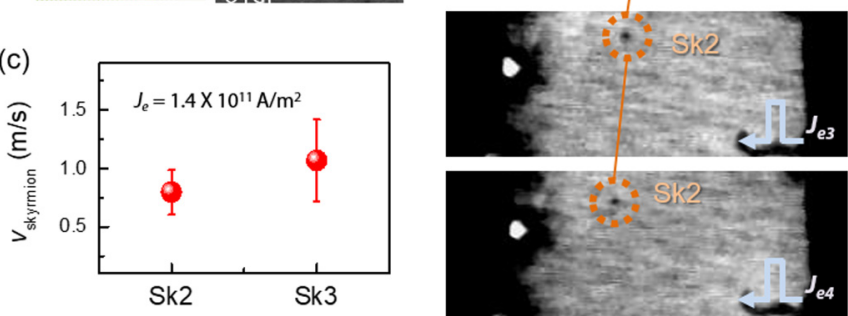

FIG. 5. Current-driven motion of skyrmions. (a) Schematic image of the FGT device used for the experiment. Two bottom inset images show the optical microscopy image of a FGT device capped by $h$-BN used for the STXM experiment (left, scale bar is 10 $\mu \mathrm{m})$, and the cross-sectional high-resolution transmission electron microscopy (HRTEM) image of the device (right, scale bar is 20 $\mathrm{nm}$ ). (b) Sequential STXM images showing skyrmion configurations in the FGT device, where each STXM image was acquired after injecting five unipolar current pulses along the $+x$ direction (electron flow to $-x$ direction), with an amplitude $J_{e}=1.4 \times 10^{11} \mathrm{~A} / \mathrm{m}^{2}$ and duration $50 \mathrm{~ns}$. All images were obtained at the oblique field of -20 $\mathrm{mT}$ and $100 \mathrm{~K}$. Individual skyrmions are outlined in colored circles for clarity (Sk 1, Sk 2, and Sk 3). Scale bar, $1 \mu \mathrm{m}$. (c) The average velocities for two representative skyrmions, Sk2 and Sk3, where error bars denote the standard deviation of multiple measurements.

described above, which confirmed the robustness of FC-driven static SkX (see Supplemental Material Fig. S7 [30]).

\section{Current-driven motion of isolated skyrmions}

To further highlight the potential of FGT-based 2D vdW heterostructures for skyrmion devices, we next demonstrate the current-driven motion of skyrmions in this material, as summarized in Fig. 5. Figure 5(a) shows a schematic image of the FGT track and electric contacts fabricated on the $\mathrm{Si}_{3} \mathrm{~N}_{4}$ membrane for STXM measurements. Note that a thin $h$-BN vdW flake is used as a capping material on FGT, where the FGT layer is sandwiched by two naturally O-FGT as was also shown in Fig. 1(b). In this experiment, we first generated the initial few-skyrmion-state [as shown in the first image of Fig. 5(b)] by applying external magnetic field to the multiskyrmion state acquired by the current-driven skyrmion generation process described in Fig. 3, while an oblique magnetic field of $B=-50 \mathrm{mT}$ (the oblique angle is $30^{\circ}$ to the zone axis) was applied at $T=100 \mathrm{~K}$. In Fig. 5(b), each image was obtained after injecting five current pulses with $J_{a}=$ $1.4 \times 10^{11} \mathrm{~A} / \mathrm{m}^{2}$ and $t_{\text {pulse }}=50 \mathrm{~ns}$. The current was applied 
along the $+x$ direction, opposite to the electron flow along the $-x$ direction as schematically indicated in each image.

It is first noteworthy that skyrmions move upon the application of current pulses, and the propagation direction is along the electron-flow direction (against current flow), where this directionality indicates that the skyrmion is driven by spin-transfer torques (STTs) arising within the FGT. This is also expected from the HRTEM image shown in Fig. 5(a), exhibiting no possible interface of FGT that could provide a vertical spin current by, e.g., SHE. As shown in Fig. 5(c), the average skyrmion velocity was measured to be $\sim 1 \mathrm{~m} / \mathrm{s}$ at a current density $J_{e}=1.4 \times 10^{11} \mathrm{~A} / \mathrm{m}^{2}$, below which no skyrmion motion is observed. The current-driven motion of skyrmion shows the potential of using skyrmions in FGT for functional device applications, such as the racetrack-type memory [20], where skyrmions act as moveable information carriers.

It should also be noted that, although we initially observed three stabilized skyrmions (Sk1-3), only one skyrmion (Sk2) remains stable and propagates along the track during the their motion. We speculate such nonideality may come from the interplay between various magnetic parameters in FGT that sharply change with temperature, which then alters skyrmion stability as was experimentally navigated in Fig. 3(c). However, we believe that further experimental studies of skyrmion motion in FGT-based 2D heterostructures using more efficient torques, e.g., SOTs, arising from some known 2D materials with very large charge-to-spin conversion efficiency, e.g., $\mathrm{WTe}_{2}$ or $\mathrm{NbSe}_{2}$, could substantially improve their electrical controllability and current-velocity relation that could exceed conventional metallic systems [45-48].

\section{E. First principle calculation on Dzyaloshinskii-Moriya interaction (DMI) from O-FGT}

With these experimental demonstrations of chiral skyrmions, their SkX state, and their current-driven motion in the FGT-based heterostructures, let us now discuss the physical origins of DMI in vdW FGT crystals. We first examined the possible DMI sources from the FGT crystal symmetry. As discussed earlier, a monolayer of FGT contains three Fe sublayers (i.e., a $2 \mathrm{D} \mathrm{Fe} \mathrm{Fe}^{\mathrm{II}}$ and Ge sublayer between two $2 \mathrm{D} \mathrm{Fe} \mathrm{Fe}^{\mathrm{III}}$ sublayers), forming a hexagonal structure that is sandwiched between two Te layers. The whole FGT monolayer structure has the noncentrosymmetric point group of $D_{3 h}[28,49]$ and thus in principle, shows no bulk DMI. Indeed, although some locally broken inversion symmetry of sublayers in FGT could result in the DMI that stabilizes Néel-type skyrmions, all possible DMI contributions in the whole FGT monolayer structure cancel each other as discussed and summarized in Supplemental Material Fig. S8 and Table S1 [30]. For example, similar to the case of the 2D hexagonal boron nitride structure with buckling [50], the top $\mathrm{Fe}^{\mathrm{III}}$ sublayer and neighboring Te layer form a lattice of $C_{3 v}$ point group with broken inversion symmetry; the interfacial DMI could be induced at the top $\mathrm{Fe}^{\mathrm{III}}$ sublayer via the superexchange along the $\mathrm{Fe}^{\mathrm{III}}-\mathrm{Te}-\mathrm{Fe}^{\mathrm{III}}$ path. However, due to the reflection symmetry of the system, the DMI contributions induced at the top and bottom $\mathrm{Fe}^{\mathrm{III}}$ sublayers are cancelled with each other and the net DMI in the whole FGT structure vanishes.

In order to elucidate the possible origin of DMI at the atomic level, we performed first principles calculations employing the approach used for multilayers comprising magnetic and heavy metals [51], oxides [52], and graphene [53] (see Methods for details [30], and Refs. [54-59] therein). We first verified that the DMI for symmetric FGT structure indeed vanishes as discussed above. For FGT crystal monolayer the calculated DMI, arising at both Fe/Te interfaces is of almost equal magnitude with opposite sign yielding negligible DMI as expected from the aforementioned crystal symmetry analysis. This is in agreement with SOC energy difference associated with the total DMI, $\Delta E_{\mathrm{SOC}}$, for the same FGT crystal monolayer presented in Fig. S9b [30]. We would also like to note that this result of nonexisting interfacial DMI in FGT bulk crystal has also been reported recently by Laref et al. [60], opposing another work by Wang et al. [24] that estimates some finite DMI within FGT.

We next investigated other possible mechanisms of induced DMI by examining global and local atomic distributions along FGT crystal and its interfaces. Figure 6(a) shows atomic concentrations across the sample acquired using the quantitative high-angle annular dark field detector (HAADF) installed in scanning transmission electron microscopy (STEM) (see Supplemental Material Fig. S2 [30] for elemental mapping images). One can note first that the concentration of each atom along the thickness of sample within in the pure FGT crystal region is symmetric and homogeneous, implying that the DMI owing to asymmetric distribution of elemental content in bulk material, as presented in Ref. [61], can be excluded in this case. However, it is noteworthy that there exists significant atomic concentration fluctuation at two FGT/O-FGT interfaces. In particular, the concentration of Te atoms at both interfaces rapidly decreases and vanishes upon oxidation, while $\mathrm{Fe}$ and $\mathrm{Ge}$ concentrations only fluctuate and recover their original values near the largest oxidation areas (oxygen peaks). Figure 6(b) shows the relative atomic concentration distribution between $\mathrm{Te}$ and $\mathrm{O}$ atoms, where their sum and difference are plotted. It becomes clearer that, while their total concentration $(\mathrm{Te}+\mathrm{O})$ fluctuates within 10-15\%, their concentration difference (Te-O) rapidly decreases from the initial bulk value of Te, $\sim 30 \%$, to its negative value, $\sim-30 \%$, around oxygen concentration peaks. This distribution variation between $\mathrm{Te}$ and $\mathrm{O}$ elemental contents strongly implies that $\mathrm{Te}$ atoms are likely substituted by $\mathrm{O}$ atoms, forming $\mathrm{Fe}_{3} \mathrm{GeTe}_{(2-x)} \mathrm{O}_{x}$ over few nanometers of oxidized interfacial regions. Furthermore, one cannot exclude the oxygen addition scenario at the interfaces either. Therefore, we performed systematic calculations of microscopic and micromagnetic DMI parameters $(d$ and $D)$, for both O-substitution and Oaddition scenarios using single crystal monolayer and bulk FGT structures [Figs. 6(c)-6(j)]. In both scenarios, we found that the DMI is anisotropic in plane yielding $d_{[100]} \neq d_{[110]}$ [Figs. 6(c)-6(i)]. Of note, similar behavior was also reported for out-of-plane magnetized bcc $\mathrm{Au} / \mathrm{Co} / \mathrm{W}$ structures [62]. For the O-substitution case, we find that the single crystal monolayer DMI is nonmonotonic as a function of oxygen concentration, being weakly anticlockwise (respectively strongly clockwise) for low (respectively high) concentrations 


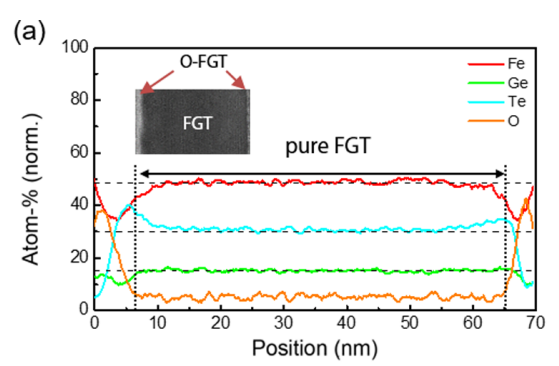

(c)

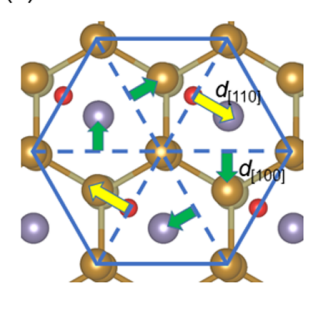

(d)

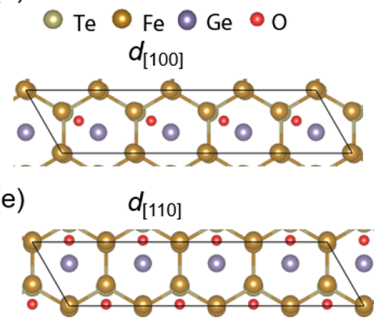

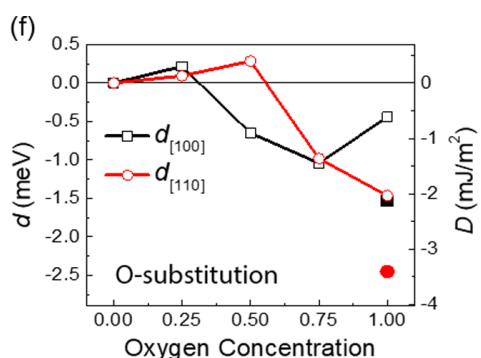

(j)

(i)

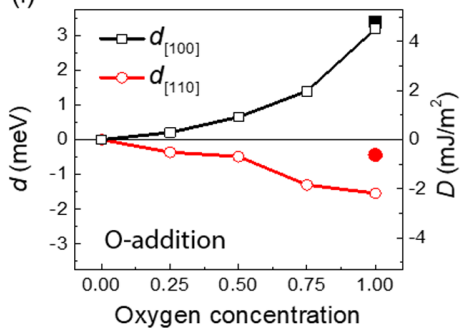

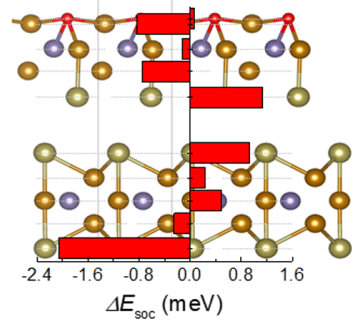

FIG. 6. First principles calculations of Dzyaloshinskii-Moriya interaction (DMI) in FGT crystal and interfaces. (a) Atomic concentration distribution of $\mathrm{Fe}, \mathrm{Ge}, \mathrm{Te}$, and $\mathrm{O}$ atoms across sample thickness within the FGT crystal used for STXM measurements. (b) Relative distribution of Te and $\mathrm{O}$ atomic concentrations, i.e., their sum and difference across the FGT sample thickness. (c) Top views of relaxed crystal of oxygen-substituted $\mathrm{Fe}_{3} \mathrm{GeTe}_{2-x} \mathrm{O}_{x}(x=1)$ relaxed structure showing DMI vectors in (d) [100] and (e) [110] directions. (f) The calculated DMI parameters in [100] and [110] in-plane directions as a function of oxygen substitution concentration of $\mathrm{Fe}_{3} \mathrm{GeTe}_{2-x} \mathrm{O}_{x}$ monolayer $(x=0$, $0.25,0.5,0.75,1)$. Solid symbols show the DMI values for O-FGT/FGT bulk structures. (g), (h) The side view of oxygen-added FGT structures along [100] and [110] directions. (i) The same as (f) for oxygen addition concentration. (j) Side view of bulk O-FGT/FGT[100] structure and layer-resolved SOC energy difference, $\Delta E_{\mathrm{soc}}$, associated with DMI distribution.

[Figs. 6(d)-6(f)]. As for the case of the O-addition scenario, the DMI strength monotonically increases as a function of oxygen concentration, although $d_{[100]}$ and $d_{[110]}$ configurations give opposite DMI chirality [Figs. 6(g)-6(i)]. Regarding the DMI in the bulk O-substituted FGT structures, very importantly, we found additional DMI contributions arising from the proximity of the pure FGT cell with the oxidized layer O-FGT. For instance, it follows from $\Delta E_{\mathrm{SOC}}$ distribution shown in Fig. 6(j) that O-FGT and FGT parts of this bulk structure provide clockwise [100] DMIs with $-0.6 \mathrm{meV}$ and $-0.8 \mathrm{meV}$ contributions, respectively, resulting in a total value of $-1.4 \mathrm{meV}$ indicated by the black solid square in Fig. 6(f). The difference of about $-1 \mathrm{meV}$ between the total DMI values for the bulk O-FGT/FGT and O-FGT fully oxidized single crystal monolayer [open square in Fig. 6(f)] clearly indicates the large clockwise DMI contribution associated with the bulk FGT part in this structure. Similar conclusions can be deduced for the [110] structure. As for the O-addition scenario, these net FGT bulk contributions in O-FGT/FGT resulting from the oxygen gradient within the structure are significantly smaller [Fig. 6(i)].

Using these theoretical findings, we can now analyze the resulting DMI in our samples supposing that these oxidation scenarios occur within interfacial areas with transient Te-O concentration represented by shaded areas in Fig. 6(b), with the top $(5.9 \mathrm{~nm})$ oxidized region being thicker compared to the bottom one $(3.3 \mathrm{~nm})$. These relatively thick regions with variable oxidation rates within them suggest that the DMI is not "localized" at narrow interfaces between atomic layers. Instead, the whole thickness regime with a finite oxidation gradient serves as a DMI-enhancing layer across a few nanometers, which works together with Heisenberg exchange, dipolar energy, and anisotropy, leading to the formation of chiral magnetic skyrmions and their lattices observed here. Although two O-FGT/FGT interfaces are symmetrically present in our FGT sample and therefore may counteract, the magnitudes of DMI in these interfaces may be very different due to a largely asymmetric oxidation profile and the aforementioned scenarios (substitution and addition). In fact, even in the case of only the substitution scenario present, the overall net clockwise DMI will be present due to oxidation region asymmetry. Moreover, both $\mathrm{O}$ and $\mathrm{Te}$ interfacial gradients favor the O-substitution scenario which gives rise to clockwise DMI provided by FGT adjacent layers.

To further corroborate our $a b$ initio study, we performed magnetic imaging of another FGT-based heterostructure without any O-FGT interfaces, as shown in Fig. S10 [30]. In this experiment, we used DPCM by configuring the magnetic structure in low-magnification scanning mode (STEM), which enables the direct measurement of the in-plane magnetic components in focus to avoid feature blurring via the defocus inherent in LTEM imaging obtained at the defocused mode [63]. The DPCM measurement and analysis present that the observed magnetic textures are Bloch type, in good agreement with previous LTEM results observed in a FGT flake without oxidized interfaces [23]. This is significant, as it highlights that we can now manipulate the chiral state of magnetic texture (i.e., Néel type vs Bloch type) in vdW heterostructures by employing different fabrication processes. Moreover, following the physical insights revealed by our work, we believe that future studies could utilize 2D heterostructures consisting of nonoxidized FGT and other oxide vdW materials (e.g., 
FGT/ $\mathrm{V}_{2} \mathrm{O}_{5}$ ) for engineering interfacial DMI in a more controllable way.

Nevertheless, we believe further systematic experimental studies probing the dependence of spin textures on the total FGT thickness, and/or the internal magnetization profile of skyrmions from top to bottom layers in FGT considering the role of van der Waals interactions could shed light into more precise tailoring of DMI and resulting magnetic textures in FGT crystal and heterostructures.

\section{CONCLUSIONS}

In summary, we observed Néel-type chiral magnetic skyrmions and their lattice phase stabilization in a vdW ferromagnet FGT using high resolution magnetic microscopy. We examined the stability of SkX in FGT over a wide range of temperatures and magnetic fields, including its zero-field manifestation. We also demonstrated current-driven motion of individual skyrmions in FGT, highlighting its potential for device applications. We performed symmetry analysis and first principles calculations to unveil the origins of the emergent Neél-type spin textures, namely DMI at the oxidized interfaces of FGT, which also demonstrates the controllability of chiral states in vdW heterostructures by process and/or interfacial material engineering. The possibility to achieve and electrically manipulate magnetic skyrmions in vdW magnets marks a significant advance in vdW magnet-based spintronics. Our results can support a further understanding of the fielddriven and current-driven dynamics of skyrmions in $2 \mathrm{D} \mathrm{vdW}$ materials and provide guidelines for the design of magnetic devices based on 2D materials. Along with the large potential of skyrmions for future spintronic devices to store, process, and transmit data with extremely low power cost, this work will pave a route towards $\mathrm{vdW}$ magnet-based topological magnetism and skyrmion electronics.

\section{ACKNOWLEDGMENTS}

S.W. acknowledges the support from IBM Research and the managemental support from G. Hu and D. Worledge. S.W. also acknowledges J. Zang for reading this manuscript and providing helpful comments. X.Z.Y. acknowledges the support from Grants-In-Aid for Scientific Research (A) (Grant No. 19H00660) from Japan Society for the Promotion of Science (JSPS). T.-E.P., S.J.K., K.M.S., K.K., J.C., and H.C.K. acknowledge the support from the KIST Institutional Program (2E31032) and the National Research Council of Science and Technology (NST) (Grant No. CAP-16-01KIST) by the Korean government (MSIP). K.K. acknowledges the support from the Basic Research Laboratory Program through the National Research Foundation of Korea (NRF) funded by the MSIT (Grant No. NRF-2018R1A4A1020696). X.Z. acknowledges the support by the National Natural Science Foundation of China (Grant No. 12004320), and the Guangdong Basic and Applied Basic Research Foundation (Grant No. 2019A1515110713). Y.Z. acknowledges the support by Guangdong Special Support Project (Grant No. 2019BT02 × 030), Shenzhen Peacock Group Plan (Grant No. KQTD20180413181702403), Pearl River Recruitment Program of Talents (Grant No. 2017GC010293), and National Natural Science Foundation of China (Grants No. 11974298 and No. 61961136006). M.E. acknowledges the support by the Grants-in-Aid for Scientific Research from JSPS KAKENHI (Grants No. JP18H03676, No. JP17K05490, and No. JP15H05854) and also the support by CREST, JST (Grants No. JPMJCR16F1 and No. JPMJCR1874). X.L. acknowledges the support by the Grants-in-Aid for Scientific Research from JSPS KAKENHI (Grants No. JP20F20363, No. 17K19074, No. 26600041, and No. 22360122). J.C. acknowledges the support of Yonsei-KIST Convergence Research Institute. Y.D.K. was supported by Samsung Research $\&$ Incubation Funding Center of Samsung Electronics under Project No. SRFC-TB1803-04 and a grant from Kyung Hee University in 2018 (Grant No. KHU-20181299). A.H. and M.C. acknowledge support from European Union's Horizon 2020 research and innovation program under Grant Agreements No. 696656 and No. 785219 (Graphene Flagship). J.L. and H.Y. acknowledge support from the National Natural Science Foundation of China (Grant No. 11874059) and Zhejiang Province Natural Science Foundation of China (Grant No. LR19A040002). Part of this work was performed at the MAXYMUS endstation at Berlin Electron Storage Ring Society for Synchrotron Radiation II (BESSYII). We thank H.Z.B. for the allocation of neutron/synchrotron radiation beamtime. Part of this work was also performed at the PolLux (X07DA) endstation of the Swiss Light Source, Paul Scherrer Institut (PSI), Villigen, Switzerland. We thank PSI for the allocation of synchrotron radiation beamtime.

S.W. designed and conceived the study. T.-E.P. prepared films, fabricated devices, and performed device characterizations with support from S.J.K. K.M.S., K.K., and Y.D.K. T.-E.P., K.M.S., K.K., M.W., S.F., J.R., and S.W. performed STXM experiments at BESSY II in Berlin, Germany and at Swiss Light Source in Villigen, Switzerland. L.P. and X.Z.Y. performed Lorentz-TEM experiments and analyzed the data. F.S.Y. performed DPCM and Lorentz-TEM experiments on nonoxidized FGT and analyzed the data. J.L., A.H., A.F., M.C., and H.Y. performed the ab initio calculations on DMI in FGT crystal, and analyzed the results. X.Z., J.X., Y.Z., M.E., and X.L. provided symmetry analysis on DMI in FGT crystal. T.-E.P. drafted and L.P., X.Z., X.Z.Y., F.S.Y., and S.W. revised the manuscript, and all authors reviewed the manuscript.
[1] A. K. Geim and I. V. Grigorieva, van der Waals heterostructures, Nature (London) 499, 419 (2013).

[2] K. S. Novoselov, A. Mishchenko, A. Carvalho, and A. H. C. Neto, 2D materials and van der Waals heterostructures, Science 353, 9439 (2016).
[3] C. Gong, L. Li, Z. Li, H. Ji, A. Stern, Y. Xia, T. Cao, W. Bao, C. Wang, Y. Wang, Z. Q. Qiu, R. J. Cava, S. G. Louie, J. Xia, and $\mathrm{X}$. Zhang, Discovery of intrinsic ferromagnetism in twodimensional van der Waals crystals, Nature (London) 546, 265 (2017). 
[4] B. Huang, G. Clark, E. Navarro-Moratalla, D. R. Klein, R. Cheng, K. L. Seyler, D. Zhong, E. Schmidgall, M. A. McGuire, D. H. Cobden, W. Yao, D. Xiao, P. Jarillo-Herrero, and $\mathrm{X} . \mathrm{Xu}$, Layer-dependent ferromagnetism in a van der Waals down to the monolayer limit, Nature (London) 546, 270 (2017).

[5] T. Song, X. Cai, M. W.-Y. Tu, X. Zhang, B. Huang, N. P. Wilson, K. L. Seyler, L. Zhu, T. Taniguchi, K. Watanabe, M. A. McGuire, D. H. Cobden, D. Xiao, W. Yao, and X. Xu, Giant tunneling magnetoresistance in spin-filter van der Waals heterostructures, Science 360, 1214 (2018).

[6] D. R. Klein, D. MacNeill, J. L. Lado, D. Soriano, E. NavarroMoratalla, K. Watanabe, T. Taniguchi, S. Manni, P. Canfield, J. Fernández-Rossier, and P. Jarillo-Herrero, Probing magnetism in 2D van der Waals crystalline insulators via electron tunneling, Science 360, 1218 (2018).

[7] Y. Deng, Y. Yu, Y. Song, J. Zhang, N. Z. Wang, Z. Sun, Y. Yi, Y. Z. Wu, S. Wu, J. Zhu, J. Wang, X. H. Chen, and Y. Zhang, Gatetunable room-temperature ferromagnetism in two-dimensional $\mathrm{Fe}_{3} \mathrm{GeTe}_{2}$, Nature (London) 563, 94 (2018).

[8] K. S. Burch, D. Mandrus, and J.-G. Park, Magnetism in twodimensional van der Waals materials, Nature (London) 563, 47 (2018)

[9] C. Gong and X. Zhang, Two-dimensional magnetic crystals and emergent heterostructure devices, Science 363, eaav4450 (2019).

[10] M. Gibertini, M. Koperski, A. F. Morpurgo, and K. S. Novoselov, Magnetic 2D materials and heterostructures, Nat. Nanotechnol. 14, 408 (2019).

[11] X. Wang, J. Tang, X. Xia, C. He, J. Zhang, Y. Liu, C. Wan, C. Fang, C. Guo, W. Yang, Y. Guang, X. Zhang, H. Xu, J. Wei, M. Liao, X. Lu, J. Feng, X. Li, Y. Peng, H. Wei et al., Currentdriven magnetization switching in a van der Waals ferromagnet $\mathrm{Fe}_{3} \mathrm{GeTe}_{2}$, Sci. Adv. 5, eaaw8904 (2019).

[12] N. D. Mermin and H. Wagner, Absence of Ferromagnetism or Antiferromagnetism in One- or Two-Dimensional Isotropic Heisenberg Models, Phys. Rev. Lett. 17, 1133 (1966).

[13] I. Dzyaloshinsky, A thermodynamic theory of "Weak" ferromagnetism of antiferromagnetics, J. Phys. Chem. Solids 4, 241 (1958).

[14] T. Moriya, Anisotropic superexchange interaction and weak ferromagnetism, Phys. Rev. 120, 91 (1960).

[15] S. Mühlbauer, B. Binz, F. Jonietz, C. Pfleiderer, A. Rosch, A. Neubauer, R. Georgii, and P. Böni, Skyrmion lattice in a chiral magnet, Science 323, 915 (2009).

[16] X. Z. Yu, Y. Onose, N. Kanazawa, J. H. Park, J. H. Han, Y. Matsui, N. Nagaosa, and Y. Tokura, Real-space observation of a two-dimensional skyrmion crystal, Nature (London) 465, 901 (2010).

[17] Q. Tong, F. Liu, J. Xiao, and W. Yao, Skyrmions in the Moiré of van Der Waals 2D magnets, Nano Lett. 18, 7194 (2018).

[18] K. M. D. Hals and K. Everschor-Sitte, Twists in ferromagnetic monolayers with trigonal prismatic symmetry, Phys. Rev. B 99, 104422 (2019)

[19] A. K. Behera, S. Chowdhury, and S. R. Das, Magnetic Skyrmions in Atomic Thin $\mathrm{CrI}_{3}$ Monolayer, Appl. Phys. Lett. 114, 232402 (2019).

[20] A. Fert, N. Reyren, and V. Cros, Magnetic skyrmions: Advances in physics and potential applications, Nat. Rev. Mater. 2, 7 (2017).
[21] X. Zhang, Y. Zhou, K. M. Song, T.-E. Park, J. Xia, M. Ezawa, X. Liu, W. Zhao, G. Zhao, and S. Woo, Skyrmion-electronics: Writing, deleting, reading and processing magnetic skyrmions toward spintronic applications, J. Phys.: Condens. Matter 32, 143001 (2020).

[22] M.-G. Han, J. A. Garlow, Y. Liu, H. Zhang, J. Li, D. DiMarzio, M. W. Knight, C. Petrovic, D. Jariwala, and Y. Zhu, Topological magnetic-spin textures in two-dimensional van der Waals $\mathrm{Cr}_{2} \mathrm{Ge}_{2} \mathrm{Te}_{6}$, Nano Lett. 19, 7859 (2019).

[23] B. Ding, Z. Li, G. Xu, H. Li, Z. Hou, E. Liu, X. Xi, F. Xu, Y. Yao, and W. Wang, Observation of magnetic skyrmion bubbles in a van der Waals ferromagnet $\mathrm{Fe}_{3} \mathrm{GeTe}_{2}$, Nano Lett. 20, 868 (2020).

[24] H. Wang, C. Wang, Z.-A. Li, H. Tian, Y. Shi, H. Yang, and J. Li, Characteristics and Temperature-Field-Thickness Evolutions of Magnetic Domain Structures in van der Waals Magnet $\mathrm{Fe}_{3} \mathrm{GeTe}_{2}$ Nanolayers, Appl. Phys. Lett. 116, 192403 (2020).

[25] Y. Wu, S. Zhang, J. Zhang, W. Wang, Y. L. Zhu, J. Hu, G. Yin, K. Wong, C. Fang, C. Wan, X. Han, Q. Shao, T. Taniguchi, K. Watanabe, J. Zang, Z. Mao, X. Zhang, and K. L. Wang, Néel-type skyrmion in $\mathrm{WTe}_{2} / \mathrm{Fe}_{3} \mathrm{GeTe}_{2}$ van der Waals heterostructure, Nat. Commun. 11, 3860 (2020).

[26] M. Yang, Q. Li, R. V. Chopdekar, R. Dhall, J. Turner, J. D. Carlström, C. Ophus, C. Klewe, P. Shafer, A. T. N'Diaye, J. W. Choi, G. Chen, Y. Z. Wu, C. Hwang, F. Wang, and Z. Q. Qiu, Creation of skyrmions in van der Waals ferromagnet $\mathrm{Fe}_{3} \mathrm{GeTe}_{2}$ on $(\mathrm{Co} / \mathrm{Pd})_{\mathrm{n}}$ superlattice, Sci. Adv. 6, eabb5157 (2020).

[27] Z. Fei, B. Huang, P. Malinowski, W. Wang, T. Song, J. Sanchez, W. Yao, D. Xiao, X. Zhu, A. F. May, W. Wu, D. H. Cobden, J.H. Chu, and X. Xu, Two-dimensional itinerant ferromagnetism in atomically thin $\mathrm{Fe}_{3} \mathrm{GeTe}_{2}$, Nat. Mater. 17, 778 (2018).

[28] H.-J. Deiseroth, K. Aleksandrov, C. Reiner, L. Kienle, and R. K. Kremer, $\mathrm{Fe}_{3} \mathrm{GeTe}_{2}$ and $\mathrm{Ni}_{3} \mathrm{GeTe}_{2}$ - two new layered transitionmetal compounds: Crystal structures, HRTEM investigations, and magnetic and electrical properties, Eur. J. Inorg. Chem. 2006, 1561 (2006).

[29] H. L. Zhuang, P. R. C. Kent, and R. G. Hennig, Strong anisotropy and magnetostriction in the two-dimensional stoner ferromagnet $\mathrm{Fe}_{3} \mathrm{GeTe}_{2}$, Phys. Rev. B 93, 134407 (2016).

[30] See Supplemental Material at http://link.aps.org/supplemental/ 10.1103/PhysRevB.103.104410 for materials, methods, and additional analysis of magnetic properties in FGT-based heterostructures.

[31] S. Woo, K. M. Song, H.-S. Han, M.-S. Jung, M.-Y. Im, K.-S. Lee, K. S. Song, P. Fischer, J.-I. Hong, J. W. Choi, B.-C. Min, H. C. Koo, and J. Chang, Spin-orbit torque-driven skyrmion dynamics revealed by time-resolved x-ray microscopy, Nat. Commun. 8, 15573 (2017).

[32] I. Lemesh, K. Litzius, M. Böttcher, P. Bassirian, N. Kerber, D. Heinze, J. Zázvorka, F. Büttner, L. Caretta, M. Mann, M. Weigand, S. Finizio, J. Raabe, M.-Y. Im, H. Stoll, G. Schütz, B. Dupé, M. Kläui, and G. S. D. Beach, Current-induced skyrmion generation through morphological thermal transitions in chiral ferromagnetic heterostructures, Adv. Mater. 30, 1805461 (2018).

[33] J. Sinova, S. O. Valenzuela, J. Wunderlich, C. H. Back, and T. Jungwirth, Spin hall effects, Rev. Mod. Phys. 87, 1213 (2015).

[34] D. Toscano, S. A. Leonel, P. Z. Coura, and F. Sato, Building traps for skyrmions by the incorporation of magnetic defects 
into nanomagnets: pinning and scattering traps by magnetic properties engineering, J. Magn. Magn. Mater. 480, 171 (2019).

[35] S. Rohart and A. Thiaville, Skyrmion confinement in ultrathin film nanostructures in the presence of dzyaloshinskii-moriya interaction, Phys. Rev. B 88, 184422 (2013).

[36] X. S. Wang, H. Y. Yuan, and X. R. Wang, A theory on skyrmion size, Commun. Phys. 1, 31 (2018).

[37] N. Nagaosa and Y. Tokura, Topological properties and dynamics of magnetic skyrmions, Nat. Nanotechnol. 8, 899 (2013).

[38] S. Woo, K. Litzius, B. Krüger, M.-Y. Im, L. Caretta, K. Richter, M. Mann, A. Krone, R. M. Reeve, M. Weigand, P. Agrawal, I. Lemesh, M.-A. Mawass, P. Fischer, M. Kläui, and G. S. D. Beach, Observation of room-temperature magnetic skyrmions and their current-driven dynamics in ultrathin metallic ferromagnets, Nat. Mater. 15, 501 (2016).

[39] M. J. Benitez, A. Hrabec, A. P. Mihai, T. A. Moore, G. Burnell, D. McGrouther, C. H. Marrows, and S. McVitie, Magnetic microscopy and topological stability of homochiral néel domain walls in a Pt/Co/AlO $x$ trilayer, Nat. Commun. 6, 8957 (2015).

[40] S. K. Walton, K. Zeissler, W. R. Branford, and S. Felton, MALTS: A tool to simulate lorentz transmission electron microscopy from micromagnetic simulations, IEEE Trans. Magn. 49, 4795 (2013).

[41] M. J. Donahue and D. G. Porter, OOMMF User's Guide, Version 1.0.

[42] W. Jiang, W. Zhang, G. Yu, M. B. Jungfleisch, P. Upadhyaya, H. Somaily, J. E. Pearson, Y. Tserkovnyak, K. L. Wang, O. Heinonen, S. G. E. te Velthuis, and A. Hoffmann, Mobile Néel skyrmions at room temperature: status and future, AIP Adv. 6, 055602 (2016).

[43] S. McVitie, S. Hughes, K. Fallon, S. McFadzean, D. McGrouther, M. Krajnak, W. Legrand, D. Maccariello, S. Collin, K. Garcia, N. Reyren, V. Cros, A. Fert, K. Zeissler, and C. H. Marrows, A transmission electron microscope study of Néel skyrmion magnetic textures in multilayer thin film systems with large interfacial chiral interaction, Sci. Rep. 8, 5703 (2018).

[44] S. D. Pollard, J. A. Garlow, J. Yu, Z. Wang, Y. Zhu, and H. Yang, Observation of stable Néel skyrmions in Cobalt/Palladium multilayers with lorentz transmission electron microscopy, Nat. Commun. 8, 14761 (2017).

[45] J. Sampaio, V. Cros, S. Rohart, A. Thiaville, and A. Fert, Nucleation, stability and current-induced motion of isolated magnetic skyrmions in nanostructures, Nat. Nanotechnol. 8, 11 (2013).

[46] D. MacNeill, G. M. Stiehl, M. H. D. Guimaraes, R. A. Buhrman, J. Park, and D. C. Ralph, Control of spin-orbit torques through crystal symmetry in $\mathrm{WTe}_{2} /$ Ferromagnet bilayers, Nat. Phys. 13, 300 (2017).

[47] M. H. D. Guimarães, G. M. Stiehl, D. MacNeill, N. D. Reynolds, and D. C. Ralph, Spin-orbit torques in $\mathrm{NbSe}_{2}$ /Permalloy bilayers, Nano Lett. 18, 1311 (2018).

[48] S. Shi, S. Liang, Z. Zhu, K. Cai, S. D. Pollard, Y. Wang, J. Wang, Q. Wang, P. He, J. Yu, G. Eda, G. Liang, and H.
Yang, All-electric magnetization switching and dzyaloshinskiimoriya interaction in $\mathrm{WTe}_{2} /$ Ferromagnet heterostructures, Nat. Nanotechnol. 14, 10 (2019).

[49] Ø. Johansen, V. Risinggård, A. Sudbø, J. Linder, and A. Brataas, Current Control of Magnetism in Two-Dimensional $\mathrm{Fe}_{3} \mathrm{GeTe}_{2}$, Phys. Rev. Lett. 122, 217203 (2019).

[50] J.-X. Yu, M. Daly, and J. Zang, Thermally driven topology in frustrated systems, Phys. Rev. B 99, 104431 (2019).

[51] H. Yang, A. Thiaville, S. Rohart, A. Fert, and M. Chshiev, Anatomy of Dzyaloshinskii-Moriya Interaction at Co/Pt Interfaces, Phys. Rev. Lett. 115, 267210 (2015).

[52] H. Yang, O. Boulle, V. Cros, A. Fert, and M. Chshiev, Controlling dzyaloshinskii-moriya interaction via chirality dependent atomic-layer stacking, insulator capping and electric field, Sci. Rep. 8, 12356 (2018).

[53] H. Yang, G. Chen, A. A. C. Cotta, A. T. N'Diaye, S. A. Nikolaev, E. A. Soares, W. A. A. Macedo, K. Liu, A. K. Schmid, A. Fert, and M. Chshiev, Significant dzyaloshinskiimoriya interaction at graphene-ferromagnet interfaces due to the rashba effect, Nat. Mater. 17, 605 (2018).

[54] G. Kresse and J. Furthmüller, Efficient iterative schemes for ab initio total-energy calculations using a plane-wave basis set, Phys. Rev. B 54, 11169 (1996).

[55] G. Kresse and J. Hafner, Ab Initio molecular dynamics for liquid metals, Phys. Rev. B 47, 558 (1993).

[56] G. Kresse and J. Hafner, Ab Initio molecular-dynamics simulation of the liquid-metal- amorphous-semiconductor transition in germanium, Phys. Rev. B 49, 14251 (1994).

[57] G. Kresse and J. Furthmüller, Efficiency of ab-initio total energy calculations for metals and semiconductors using a plane-wave basis set, Comput. Mater. Sci. 6, 15 (1996).

[58] J. P. Perdew, K. Burke, and M. Ernzerhof, Generalized Gradient Approximation Made Simple, Phys. Rev. Lett. 77, 3865 (1996).

[59] H. J. Xiang, E. J. Kan, S.-H. Wei, M.-H. Whangbo, and X. G. Gong, Predicting the spin-lattice order of frustrated systems from first principles, Phys. Rev. B 84, 224429 (2011).

[60] S. Laref, K.-W. Kim, and A. Manchon, Elusive dzyaloshinskiimoriya interaction in monolayer $\mathrm{Fe}_{3} \mathrm{GeTe}_{2}$, Phys. Rev. B 102, 060402 (2020).

[61] D.-H. Kim, M. Haruta, H.-W. Ko, G. Go, H.-J. Park, T. Nishimura, D.-Y. Kim, T. Okuno, Y. Hirata, Y. Futakawa, H. Yoshikawa, W. Ham, S. Kim, H. Kurata, A. Tsukamoto, Y. Shiota, T. Moriyama, S.-B. Choe, K.-J. Lee, and T. Ono, Bulk dzyaloshinskii-moriya interaction in amorphous ferrimagnetic alloys, Nat. Mater. 18, 685 (2019).

[62] L. Camosi, S. Rohart, O. Fruchart, S. Pizzini, M. Belmeguenai, Y. Roussigné, A. Stashkevich, S. M. Cherif, L. Ranno, M. de Santis, and J. Vogel, Anisotropic dzyaloshinskii-moriya interaction in ultrathin epitaxial $\mathrm{Au} / \mathrm{Co} / \mathrm{W}(110)$, Phys. Rev. B 95, 214422 (2017).

[63] S. McVitie and J. N. Chapman, Reversal mechanisms in lithographically defined magnetic thin film elements imaged by scanning transmission electron microscopy, Microsc. Microanal. 3, 146 (1997). 